\title{
DIGITALCOMMONS
}

\section{Applying Spatial Randomness To Community Inclusion}

\author{
Michael Wolf-Branigin \\ Caliber Associates, Fairfax, VA
}

Follow this and additional works at: http://digitalcommons.wayne.edu/jmasm

Part of the Applied Statistics Commons, Social and Behavioral Sciences Commons, and the Statistical Theory Commons

\section{Recommended Citation}

Wolf-Branigin, Michael (2002) "Applying Spatial Randomness To Community Inclusion," Journal of Modern Applied Statistical Methods: Vol. 1 : Iss. 1 , Article 14.

DOI: $10.22237 /$ jmasm/1020255300

Available at: http://digitalcommons.wayne.edu/jmasm/vol1/iss1/14 


\title{
Applying Spatial Randomness To Community Inclusion
}

\author{
Michael Wolf-Branigin \\ Caliber Associates \\ Fairfax, VA
}

A spatial analytic methodology incorporating true locations is demonstrated using Monte Carlo simulations as a complement to current psychometric and quality of life indices for measuring community inclusion. Moran's $I$, a measure of spatial autocorrelation, is used to determine spatial dependencies in housing patterns for multiple variables, including family/friends involvement in future planning, home size, and earned income. Simulations revealed no significant spatial autocorrelation, which is a socially desirable result for housing locations for people with disabilities. Assessing the absence of clustering provides a promising methodology for measuring community inclusion.

Keywords: Spatial analysis, Monte Carlo methods, Community inclusion, Spatial randomness

\section{Introduction}

During the past decades employment, housing, educational policies, and other services for people with developmental disabilities have shifted to community settings in the United States. Self-determination, individualized budgets, and consumer-focused planning techniques are rapidly gaining influence within the disability field as people with disabilities are encouraged to explore and connect with their community, seek new experiences, learn how to live, work, recreate, and go to school with non-disabled peers (Mount, 1987; Rhodes, McFarland, \& Knight, 1995). This has resulted in more people with disabilities who work and live in increasingly more independent employment and housing settings (Braddock, Hemp, Parish, Westrich, \& Park, 1997). The objective has been to provide improved and relevant services in a fully integrated environment.

Spatial analysis, a methodology for assessing patterns, networks, and interactions, is frequently used to measure access to services for the general public by urban planners and geographers. However, the potential benefits of spatial analysis have yet to be considered by human service professionals to assess accessibility and inclusion for people with developmental disabilities. The use of spatial data as a component of social program evaluation practice is easily suggested by the fact that approximately $80 \%$ of human service organization databases have some locational element included (Hutchinson \& Daniel, 1995).

Spatial analyses might lead to increased understanding of relationships, and the degree to which people with disabilities interact with their environment. An example would be a study of community inclusion for people

Michael Wolf-Branigin is Senior Associate, Caliber Associates, 10530 Rosehaven Street, Suite 400, Fairfax, VA, 22030. His research activities are in substance abuse treatment, education policy for children with special needs, consumer-choice models, and disability and health. He is an accreditation surveyor with CARF, The Rehabilitation Accreditation Commission. with developmental disabilities who have moved from institutional or segregated type housing to community settings. These community settings include small group homes, semi-independent living, or independent living apartments.

\section{Purpose of the Study}

The purpose of this article is to introduce spatial analytic methods that compare true housing locations against randomly generated patterns using Monte Carlo simulations. The results of the simulations will be used to complement quality of life, psychometric, and attitudinal scales for examining levels of community inclusion and social interactions. This leads to the primary research question: Does spatial analysis, specifically a random pattern of location (i.e., lack of spatial autocorrelation) as identified through Moran's I, provide a useful method for measuring physical community inclusion for people with developmental disabilities?

Several explanatory variables (percentage of earned income to total income, size of the individual's support system, level of disability, and number of people with whom the individual lives who also have a disability) will be used to identify spatial dependencies. For example, do people with disabilities who have some earned income have a greater level of community inclusion than people who only receive income from governmental sources? This quantification of location allowed spatial features to be geographically referenced and mapped. Concurrently quantification of attributes facilitates the identification and analysis of spatial relationships.

With the greater adoption of community inclusion and normalization principles in the human service delivery sector, people with developmental disabilities are increasingly finding housing options in their communities. This and similar shifts of human services from a centralized service providers to more individualized approaches has created the need for evaluative strategies focusing on 
environmental interactions. Decentralized services, such as housing patterns of people with developmental disabilities, are a goal of community inclusion.

\section{Moran's I}

Within this community inclusion context, the absence of spatial autocorrelation (SAC) will be desirable. That means there should be no interaction or dependence between points (Diggle, 1983). The ideal pattern has been referred to as complete spatial randomness (CSR).

The test statistic, Moran's I, measures the degree of spatial autocorrelation (Cressie, 1993). It can be decomposed from an overall value to individual observations for each case (Anselin, 1995a). These decomposed values are referred to as local indicators of spatial association (LISAs). Point pattern analysis was used because of the additional advantage of requiring only location and attribute data for analysis.

Moran's I requires interval-scale observations and is useful in describing the spatial patterns of distributions. It is essentially a Pearson product-moment correlation coefficient modified to account for a spatial weight matrix (Getis, 1991). Values range from -1.0 to 1.0. A high value (approaching 1.0) indicates a clustered pattern, while a low value (approaching -1.0 ) indicates a scattered pattern. A value near zero indicates randomness. As individuals become more included in their communities the desire is for the patterns to approximate zero $(0.0)$.

Method

Data were obtained for individuals who currently reside in a variety of community-based settings. The types of homes include supported living, shared lives, foster and adoptive families, and small group homes. Data were also collected on the number of non-related people (with disabilities) who live in the same home.

Data were collected for eighty-six randomly selected individuals. The desired sample size was derived from Monte Carlo studies by Anselin \& Keleijian (1997), who suggested that samples of at least $\mathrm{N}=81$ with normally distributed error terms achieves the asymptotic form of Moran's I test, based on independent variable residuals. The same study indicated that the test possesses good overall power, but may under-reject the null hypothesis when it is in fact true with a smaller sample $(\mathrm{N}=48)$. This sample size for the autocorrelation analysis is also consistent with Cohen (1992) for medium effect sizes of . 30 and .15, respectively.

The physical inclusion of people in their communities was assessed using a variety of spatial analytic approaches centering on point pattern analysis. Independent variables included the degree to which people with disabilities had involvement of family, friends and allies in planning their futures; number of people with disabilities residing in their home; and the level of individual earned income. included:

Following random selection, research procedures

1. collecting data at the individual consumer level,

2. mapping of this data in a two dimensional space,

3. performing autocorrelation analysis of housing locations using Moran's I,

4. creating local indicators of spatial association (LISA) for use as dependent variable, and

5. generating 999 random permutations for assessing pseudo-significance.

Data were collected from agency records and a review of annual planning meeting records. Data concerning the income types and levels for the income records was obtained from the current records of the consumer located in the medical records office of the organization.

Monte Carlo simulations were performed with SpaceStat software (Anselin, 1995b). Developing local indicators of spatial association (LISA) as the dependent variable required the creation of a spatial weights matrix using SpaceStat software. Statistics calculated included Moran's I for the distance bands indexes. Simulations for assessing the robustness of the Moran's I were conducted. This method followed Yung and Chan's (1999) three-step procedure, including defining a pseudo-population, resampling, and evaluation. Runs of 999 drawn from the sample were performed for the exploratory variables that were believed to be potentially spatially autocorrelated.

\section{Results}

Descriptive Statistics

Descriptive statistics are summarized below. The most common size of household was six persons (36.9\%). Twelve (14.3\%) individuals resided alone in independent or semi-independent housing. The proportion of earned income was highly variable as demonstrated by the standard deviation (.1591) being approximately twice the mean (.07639). Having one non-professional person at the planning meeting was the most frequent (45.2\%) followed by having two people present $(28.6 \%)$. Males and females comprised $56 \%(n=47)$, and $44 \%(n=37)$, respectively of the sample. Many people needed assistance with mobility. This was defined as the person not being able to drive, transport self to daily activities or use public transportation. Forty-eight $(57.14 \%)$ did not need assistance while $36(42.86 \%)$ did. 
Table 1. Autocorrelation for Distance Band Measures

\begin{tabular}{|l|c|c|c|c|}
\hline Explanatory Variable & Moran's I & Mean & Standard Deviation & Probability \\
\hline & & & & \\
Proportion of earned income & -.019 & -.010 & .0331 & .436 \\
Size of Home & .032 & -.012 & .0368 & .330 \\
People at Planning Meeting & -.014 & -.014 & .0364 & .250 \\
Level of Disability & -.013 & -.013 & .0353 & .222 \\
Mobility & -.013 & -.013 & .0344 & .123 \\
\hline
\end{tabular}

Note: Empirical significance based on 999 random permutations.

\section{Spatial Analyses}

Monte Carlo procedures using Moran's I spatial autocorrelation statistics for distance band measures did not reveal significant clustering for any of the exploratory variables. See Table 1 . The distance band figures for proportion of earned income were $(I=-.019 ; p=.436)$, home size $(I=-.032 ; p=.330)$, number of people at the person's annual planning meeting $(\mathrm{I}=-.014 ; \mathrm{p}=.250)$, level of disability $(\mathrm{I}=-.013 ; \mathrm{p}=.222)$, and level of mobility $(\mathrm{I}=$ $-.013 ; \mathrm{p}=.123)$ respectively. The values for Moran's I were small (between -.019 and .032), thus indicating the absence of significant spatial autocorrelation. This suggests that the housing pattern as measured through spatial autocorrelation as a construct of complete spatial randomness was random.

\section{Conclusion}

A goal of this research was to demonstrate that spatial analysis (and more specifically point pattern analysis) methods incorporating true observations with Monte Carlo simulations provides a potentially useful tool for organizations providing housing services to people with developmental disabilities. Although measuring autocorrelation through complete spatial randomness (CSR) may be a goal of inclusion in an ideal situation, certain restraints such as access to public transportation may make this goal unrealistic. Therefore, evaluations at the local organizational level must be aware and sensitive to these exigencies. Conversely, some level of clustering may be a reasonable response to meet these demands.

Spatial analysis appears promising for measuring the degree that physical inclusion of people with developmental disabilities occurs in their communities. The results address the degree to which people were dispersed geographically throughout their community. Further application of these spatial methods could focus on issues prevalent in the current body of literature, including: the modifiable areal (or geographic) unit problem (MAUP), sensitivity issues when measuring households versus individuals, integration of spatial statistics with geographical information systems, and improved identification of inclusion through implementing a network analysis approach. Investigating autocorrelation through Monte Carlo simulations could be simplified by using all the addresses of housing locations maintained by an organization. Having a sample with multiple people residing at one location lead to statistical coding problems. Simulations with the current data set using two spatial software packages (SpaceStat and Systat 9.0 Spatial Statistics) were problematic due to co-located data. Using the addresses only once in the data collection process and analysis would eliminate this problem. Weights for the number of people at each location could be calculated into the analysis.

Paramount to understanding the logic of spatial analysis is to realize that it is an inductive process. Exploratory and interactive spatial analysis often relies on induction as opposed to a deductive hypothesis testing approach. This study provided initial support for the use of Monte Carlo simulations in spatial point pattern.

\section{References}

Anselin, L. (1995a). Local indicators of spatial association - LISA. Geographical Analysis 27, 2:93-115.

Anselin, L. (1995b). SpaceStat Version 1.80 User's Guide. Regional Research Institute, West Virginia University.

Anselin, L., \& Kelejian, H. (1997). Testing for spatial error autocorrelation in the presence of endogenous regressors. International Regional Science Review, 20, 1\&2. Regional Research Institute. University of West Virginia.

Braddock, D., Hemp, R., Parish, S., Westrich, J., \& Park, H. (1997). The State of the States in Developmental Disabilities (5th ed.). Washington, D.C. AAMR. 
Chou, Y-H. (1997). Exploring spatial analysis in geographic information systems. Santa Fe: Onward Press.

Cohen, J. (1992). A power primer. Psychological Bulletin, 112, 155-159.

Cressie, N. (1993). Statistics for spatial data. New York: Wiley.

Diggle, P. (1983). Statistical analysis of spatial point patterns. New York, Academic Press.

Gettis, A. (1991). Spatial interaction and spatial autocorrelation: A cross-product approach. Environment and Planning, $A$ 23, 1269-1277.

Hutchinson, S., \& Daniel, L. (1995). Inside ArcView. Santa Fe: Onward Press.

Mount, B. (1987). Personal futures planning: Finding directions for change (Doctoral dissertation, University of Georgia, 1987). University Microfilms International, Order No. 8714642.

Rhodes, D., McFarland, K., \& Knight, P. (1995). Evolution of consumerism in Rehabilitation counseling: A theoretical perspective. Journal of Rehabilitation, 61 (3).

Systat 9.0 Statistics II (1999). Chicago: SPSS, Inc.

Yung, Y-F., \& Chan, W. (1999). Statistical analyses using bootstrapping: Concepts and implementation. In (R. H. Hoyle, ed.) Statistical strategies for small sample research. Thousand Oaks, CA: Sage.

$$
\begin{gathered}
\text { Appendix } \\
\text { Moran's I Formula } \\
I=\frac{n \Sigma_{i} \Sigma_{j} \delta_{i j}\left(x_{i}-\bar{x}\right)\left(x_{j}-\bar{x}\right)}{S_{0} \Sigma_{i}\left(x_{i}-x\right)^{2}}
\end{gathered}
$$

where $S_{o}=\Sigma_{i} \Sigma_{j} \delta_{i j}$.

The expected value for the statistic is

$$
E(l)=-(n-1)^{-1}
$$

with the variance being

$$
\operatorname{Var}(I)=\frac{n^{2} S_{1}-n S_{2}+3 S_{0}^{2}}{S_{0}^{2}\left(n^{2}-1\right)}
$$

where

$$
S_{1}=(1 / 2) \Sigma_{i} \Sigma_{j} \quad\left(\delta_{i j}+\delta_{j i}\right)^{2}
$$

and

$$
S_{2}=\Sigma_{i}\left(\Sigma_{i} \delta_{i j}+\Sigma_{j} \delta_{j i}\right)^{2}
$$

The number of geographic units is represented by $n$, The spatial relationship between the $i$-th and $j$-th units is $\delta_{i}$, the frequency of the spatial phenomenon is $x_{p}$, and $\mathrm{S}$ ${ }_{0}$ being the number of pairs in the spatial relationship (Chou, 1995). 